\title{
Temperature variations in Titan's upper atmosphere: Impact on Cassini/Huygens
}

\author{
B. Kazeminejad ${ }^{1}$, H. Lammer ${ }^{1}$, A. Coustenis ${ }^{2}$, O. Witasse ${ }^{3}$, G. Fischer ${ }^{1}$, K. Schwingenschuh ${ }^{1}$, A. J. Ball ${ }^{4}$, and \\ H. O. Rucker ${ }^{1}$ \\ ${ }^{1}$ Space Research Institute, Austrian Academy of Sciences, A-8042 Graz, Austria \\ ${ }^{2}$ LESIA-Observatoire de Paris-Meudon, 92195, Meudon Cedex, France \\ ${ }^{3}$ ESA Research and Scientific Support Department, ESTEC, 2200 AG Noordwijk, The Netherlands \\ ${ }^{4}$ Planetary and Space Sciences Research Institute, The Open University, Milton Keynes, MK7 6AA, UK
}

Received: 5 October 2004 - Revised: 11 February 2005 - Accepted: 16 February 2005 - Published: 3 June 2005

\begin{abstract}
Temperature variations of Titan's upper atmosphere due to the plasma interaction of the satellite with Saturn's magnetosphere and Titan's high altitude monomer haze particles can imply an offset of up to $\pm 30 \mathrm{~K}$ from currently estimated model profiles. We incorporated these temperature uncertainties as an offset into the recently published Vervack et al. (2004) (Icarus, Vol. 170, 91-112) engineering model and derive extreme case (i.e. minimum and maximum profiles) temperature, pressure, and density profiles. We simulated the Huygens probe hypersonic entry trajectory and obtain, as expected, deviations of the probe trajectory for the extreme atmosphere models compared to the simulation based on the nominal one. These deviations are very similar to the ones obtained with the standard Yelle et al. (1997) (ESA SP-1177) profiles. We could confirm that the difference in aerodynamic drag is of an order of magnitude that can be measured by the probe science accelerometer. They represent an important means for the reconstruction of Titan's upper atmospheric properties. Furthermore, we simulated a Cassini low Titan flyby trajectory. No major trajectory deviations were found. The atmospheric torques due to aerodynamic drag, however, are twice as high for our high temperature profile as the ones obtained with the Yelle maximum profile and more than 5 times higher than the worst case estimations from the Cassini project. We propose to use the Cassini atmospheric torque measurements during its low flybys to derive the atmospheric drag and to reconstruct Titan's upper atmosphere density, pressure, and temperature. The results could then be compared to the reconstructed profiles obtained from Huygens probe measurements. This would help to validate the probe measurements and decrease the error bars.
\end{abstract}

Keywords. Atmospheric composition and structure (pressure, density and temperature) - Ionosphere (ionosphere magnetosphere interactions: Instruments and techniques)

Correspondence to: $\mathrm{B}$. Kazeminejad

(Bobby.Kazeminejad@rssd.esa.int)

\section{Introduction}

Solar occultation and airglow data obtained by the Voyager 1 Ultraviolet Spectrometer (UVS) during the November 1980 flyby of Titan are the primary sources of information we have on Titan's upper atmosphere. Solar occultation data consist of spectra taken before and during atmospheric attenuation, from which the line-of-sight optical depth of the observed material is determined. From the optical depth information, number densities for the various species can be inferred and the corresponding scale heights of the density profiles yield temperatures. The altitude dependent temperature profile of Titan's thermosphere is driven by two principal energy sources, solar X-ray and extreme ultraviolet (XUV) radiation and magnetospheric particles (i.e. electrons and ions). Lellouch et al. (1990) and Müller-Wodarg and Yelle (2002) calculated the diurnal variation of the vertical structure of Titan's thermosphere by using solar XUV heating, low-energy magnetospheric electron precipitation and IR cooling. Yelle (1991) provided a physical model for the thermal structure of the upper atmosphere on the basis of the calculation of radiative-conductive equilibrium temperature profiles. Those were then replaced by the empirical functions in the Yelle et al. (1997) Titan engineering atmospheric model, which is used as the nominal model for the trajectory analysis of the ESA Huygens probe, which will land on Titan on 14 January 2005 (Lebreton and Matson, 2002).

Recently, Vervack et al. (2004) reanalyzed the Voyager 1 UVS solar occultation data of Titan's upper atmosphere to expand on previous analysis. The authors present a new engineering model which is consistent with their reanalysis results in the upper atmosphere, merges smoothly with the Yelle et al. (1997) model in the lower atmosphere, and does not have a mesosphere.

Titan's upper atmosphere conditions will influence both the Huygens probe entry trajectory (Kazeminejad et al., 2004), as well as all of the currently planned ${ }^{1} 21$ targeted

\footnotetext{
${ }^{1} 041210$ Cassini reference trajectory
} 


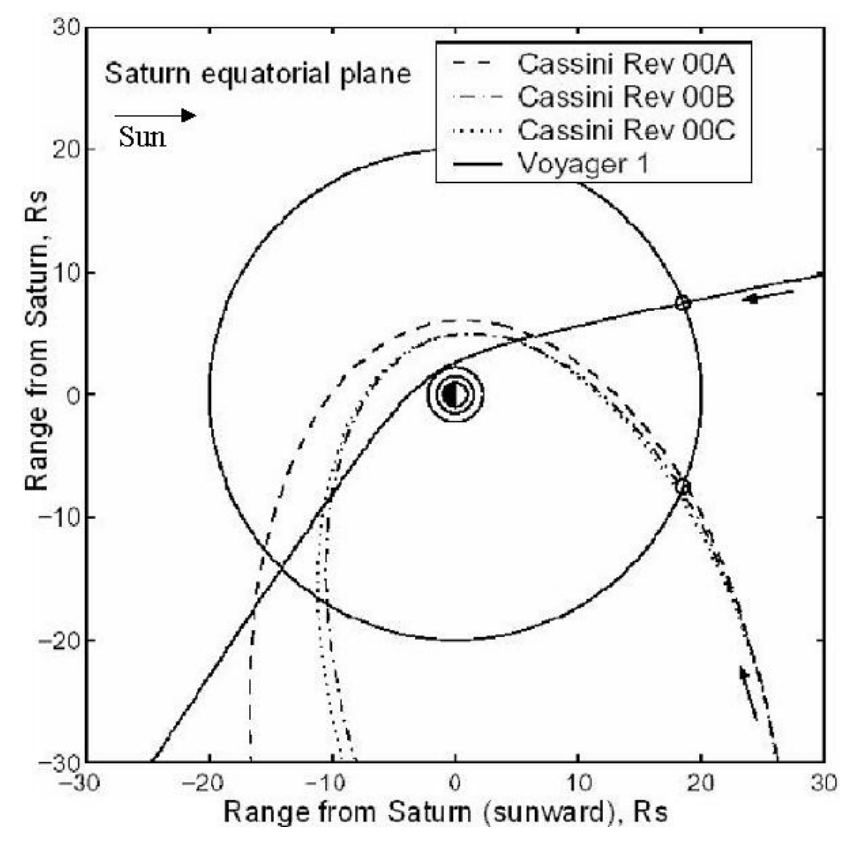

Fig. 1. Voyager 1 Titan flyby and Cassini's first three orbits. The Cassini/Huygens data relay occurs on 14 January 2005 (around 09:00 UTC=10:30 Saturn LT). The Voyager 1 flyby occured around 13:30 Saturn LT (12 November 1980).

Cassini low Titan flybys which go down to an altitude of $\sim 1000 \mathrm{~km}$ (N. Strange, private communication). We evaluate the impact of temperature uncertainties in Titan's upper atmosphere on the atmospheric drag acting on both Huygens and Cassini. For the sake of simplicity we constrain ourselves to the Cassini T16 flyby on 22 July 2006. The aim of our investigation is to conclude whether the different temperature conditions in Titan's upper atmosphere cause major deviations on the Huygens and Cassini trajectories, and if not, to assess whether they are significant enough to be sensed by the onboard accelerometers.

We review the possible heating mechanisms in Titan's upper atmosphere in Sect. 2 and explain how we incorporated them into the existing atmosphere models (see Sect. 3). We propose two new temperature and corresponding density profiles which are then ingested into our numerical simulations of the Huygens entry and Cassini flyby trajectories (see Sect. 4 and Sect. 5, respectively).

\section{Atmosphere heating mechanisms}

Titan's orbital radius of 20.2 Saturn radii is such that the satellite may be located in the solar wind, in Saturn's magnetosheath or its magnetosphere. In either of these cases different particle populations will be present and can act as sputtering agents and affect the upper atmospheric temperature profile. The present observational knowledge of Titan's atmospheric interaction with the surrounding plasma flow is based on the data from one single encounter of the Voyager 1 spacecraft in 1980. At that time Titan was located inside
Saturn's magnetosphere (e.g. Hartle et al., 1982). A loss rate of $\sim 10^{24}$ heavy $\mathrm{N}^{+}$or $\mathrm{N}_{2}^{+} / \mathrm{H}_{2} \mathrm{CN}^{+}$ions s${ }^{-1}$ has been estimated from the observations (Neubauer et al., 1984). A part of these escaping heavy atmospheric species form a nitrogen torus at Titan's orbit and contribute after ionization to Saturn's magnetospheric plasma environment. One has therefore two different incident particle populations: protons with energies of $\sim 210 \mathrm{eV}$ and $\mathrm{N}^{+}$ions with average energies of $\sim 2.9 \mathrm{keV}$ (Neubauer et al., 1984) and when Titan is outside Saturn's magnetosphere, solar wind protons with energies on the order of $\sim 1 \mathrm{keV}$ act as incident particles. Because Titan does not possess an intrinsic magnetic field, magnetospheric ions can penetrate below Titan's exobase where they act again as sputter agents (Lammer and Bauer, 1993; Shematovich et al., 2003) and can contribute to the heating of the upper atmosphere.

Lammer et al. (1998), Lammer and Bauer (1993), and Shematovich et al. (2003) used the plasma parameters observed by the Voyager 1 flyby and showed that incident $\mathrm{N}^{+}$ions are responsible for an atmospheric mass loss due to sputtering on the order of about $0.4-7.0 \times 10^{26} \mathrm{~s}^{-1}$. The bulk of incoming energetic particles are deposited below the exobase at an altitude of $\sim 1000 \mathrm{~km}$ and change the character of Titan's upper atmosphere (Lammer et al., 1998). Energetic $\mathrm{N}^{+}$ions are absorbed above an altitude of $800 \mathrm{~km}$ and one can therefore neglect the cooling process due to nonlocal thermospheric equilibrium (NLTE) by minor constituents, which is considered as the main cooling process below $800 \mathrm{~km}$ (Friedson and Yung, 1984). Lammer et al. (1998) modelled the magnetospheric plasma heating and the resulting rise in temperature above $1000 \mathrm{~km}$ in Titan's upper atmosphere by solving a time-dependent heat equation and an estimation of temperature reduction by horizontal winds to the nightside caused by pressure gradients. Their model shows that sputtering induced heating of Titan's upper atmosphere by magnetospheric $\mathrm{N}^{+}$ions may cause a temperature effect up to $\sim 30 \mathrm{~K}$ above $1000 \mathrm{~km}$ of altitude. It should be noted that the additional heating effects depend on the flux of penetrating ions, diffusion cross sections and Saturn's magnetopause location related to solar wind activity. If particle heating was also involved during the Voyager 1 flyby, the estimated temperature may be up to $30 \mathrm{~K}$ cooler compared to a situation when Titan is in the solar wind or the incident $\mathrm{N}^{+}$flux changes along the orbit. On the other hand, higher $\mathrm{N}^{+}$flux values may raise the temperature in Titan's upper atmosphere. Titan's orbital location during the first Cassini flybys (i.e. Ta, Tb) and during the Huygens mission (i.e. Tc) differs from that during the Voyager 1 flyby in 1980 (see Fig. 1). One can therefore expect widely varying and different plasma conditions in the incident flow (Wolf and Neubauer, 1982). These can result in different heating effects and imply different temperature and density profiles in the upper atmosphere.

An additional heat source in Titan's atmosphere is high altitude haze. Previous studies by Chassefière and Cabane (1995) and Lammer and Stumptner (1999) showed that monomer haze particles absorb solar radiation, emit in the infrared, and are energetically linked to the surrounding gas 
by thermal conduction. These high altitude monomers may therefore affect Titan's thermosphere profile by up to $20 \mathrm{~K}$ in an altitude range from $500-800 \mathrm{~km}$. This depends on the formation altitude of fluffy non-spherical aggregates, the monomer size, and their distribution.

\section{Titan atmosphere engineering models}

Three atmosphere models are so far available for Titan. The LH90 model (Lellouch et al., 1990; Lellouch and Hunten, 1987 ) is based on a reanalysis of the radio occultation measurements of Lindal et al. (1983) for the altitudes lower than $200 \mathrm{~km}$. The Y97 model (Yelle et al., 1997) is also based on the radio occultation data from Lindal et al. (1983) but is further constrained by Voyager 1 IRIS measurements (Coustenis et al., 1989; Coustenis and Bezard, 1995; Lellouch et al., 1989,1990 ) and, at altitudes above $\sim 1000 \mathrm{~km}$, by the UVS solar occultation experiment from Smith et al. (1982) and Strobel et al. (1992). In order to predict the physical properties of Titan's atmosphere and provide an engineering model for the Huygens project mission analysis efforts, Yelle et al. (1997) considered the following three uncertainties: uncertainties in the analysis of the Voyager data, temporal and/or spatial (i.e. latitudinal) variations of the atmospheric structure and composition that would impact the atmosphere's temperature profile, and finally, variations in the surface pressure due to topography and/or weather systems. To take into account these three categories of uncertainties Yelle et al. (1997) provided three versions of the Y97 model: a recommended model, a model for maximum mass density, and a model for minimum mass density. Note that the minimum and maximum model have a temperature difference of roughly $30 \mathrm{~K}$.

Recently, Vervack et al. (2004) reanalyzed the Voyager 1 UVS solar occultations by Titan to expand upon previous analysis and to resolve inconsistencies that have been noted in the scientific literature. The proposed V04 engineering atmosphere model assumes an atmosphere composed of $\mathrm{N}_{2}$ and $\mathrm{CH}_{4}$ and is well-mixed at all altitudes (i.e. no diffusive separation). Below the reference "surface" level at $330 \mathrm{~km}$, the V04 model is identical to the Y97 model. The most striking difference with respect to the Y97 model is the lack of a mesosphere. Vervack et al. (2004) provides two temperature profiles, one based on the UVS ingress and the other on the UVS egress occultation data. We adopted the simple engineering equation of Vervack et al. (2004) to simulate the V04 ingress temperature profile. Furthermore we introduced a minimum and maximum V04 profile with a constant temperature offset of $\pm 30 \mathrm{~K}$ to take into account the possible heating effects by magnetospheric $\mathrm{N}^{+}$ions and high altitude haze (see Sect. 2) and to join the minimum and maximum Y97 profiles below $330 \mathrm{~km}$. The upper panel of Fig. 2 depicts the temperature profiles of the three Y97 profiles and the corresponding V04 (ingress) profiles. The shaded areas show regions where the V04 temperature profiles exceed the envelope of the Y97 minimum and maximum profiles. The lower
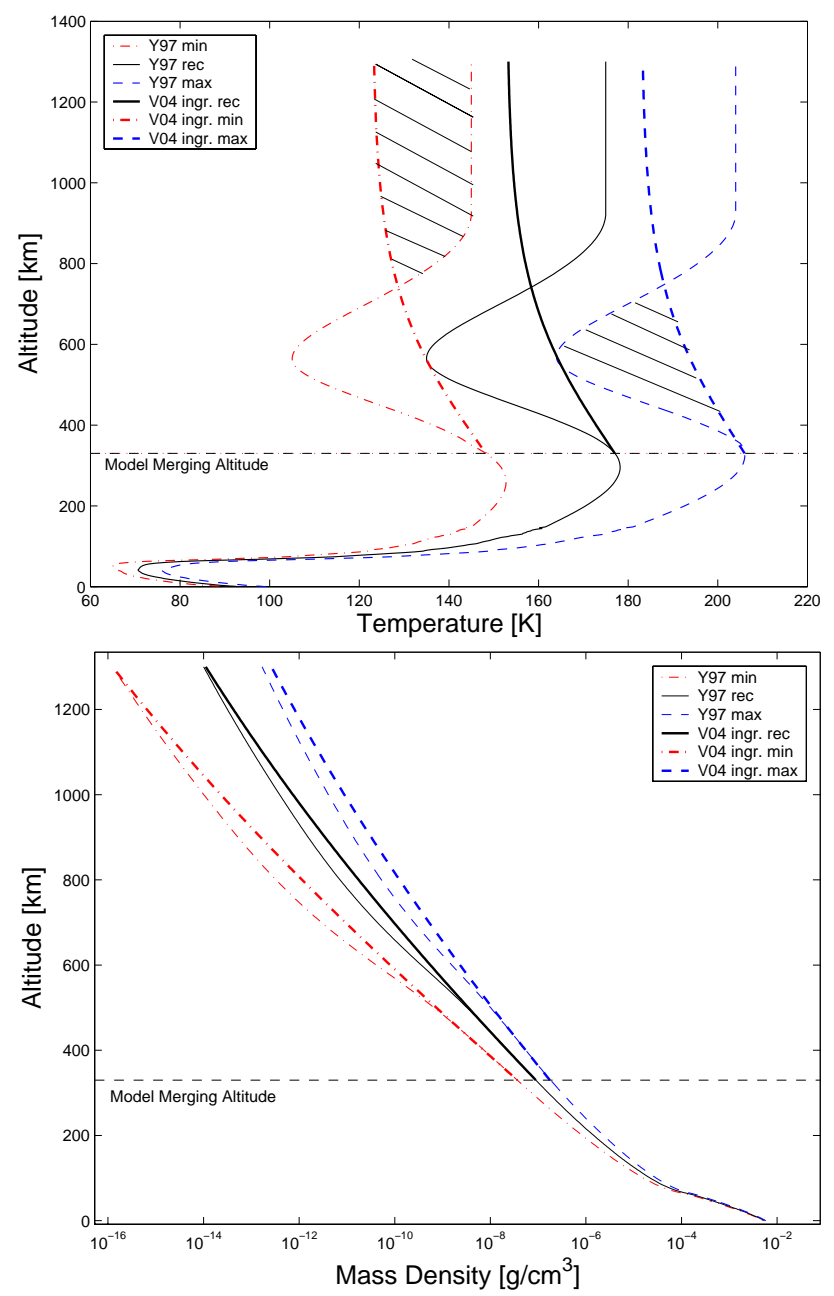

Fig. 2. Comparison of the Y97 and the V04 temperature (upper panel) and density (lower panel) profiles. The profiles merge at an altitude of $330 \mathrm{~km}$, as depicted by the horizontal line. The shaded areas in the upper panel correspond to the areas where the V04 temperature values exceed the Y97 minimum and maximum profiles.

panel of Fig. 2 shows the corresponding derived density profiles. One can see that the different thermosphere gradient and the lower V04 temperatures at $1300 \mathrm{~km}$ implies higher densities for the V04 model compared to the Y97 profiles. In the subsequent sections we will apply the three versions of the V04 model to our trajectory simulations of both the Huygens probe during its entry phase and Cassini during its low flyby on T3.

\section{Huygens entry simulations}

The Huygens probe entry trajectory spans from the defined interface altitude of $\sim 1270 \mathrm{~km}$ down to the initiation of the parachute sequence at nominally $\sim 158 \mathrm{~km}$. During this phase the probe is protected from the atmospheric induced radiative and convective heat fluxes by a $2.7-\mathrm{m}$ diameter front heat shield as it decelerates from about Mach 22.5 to 

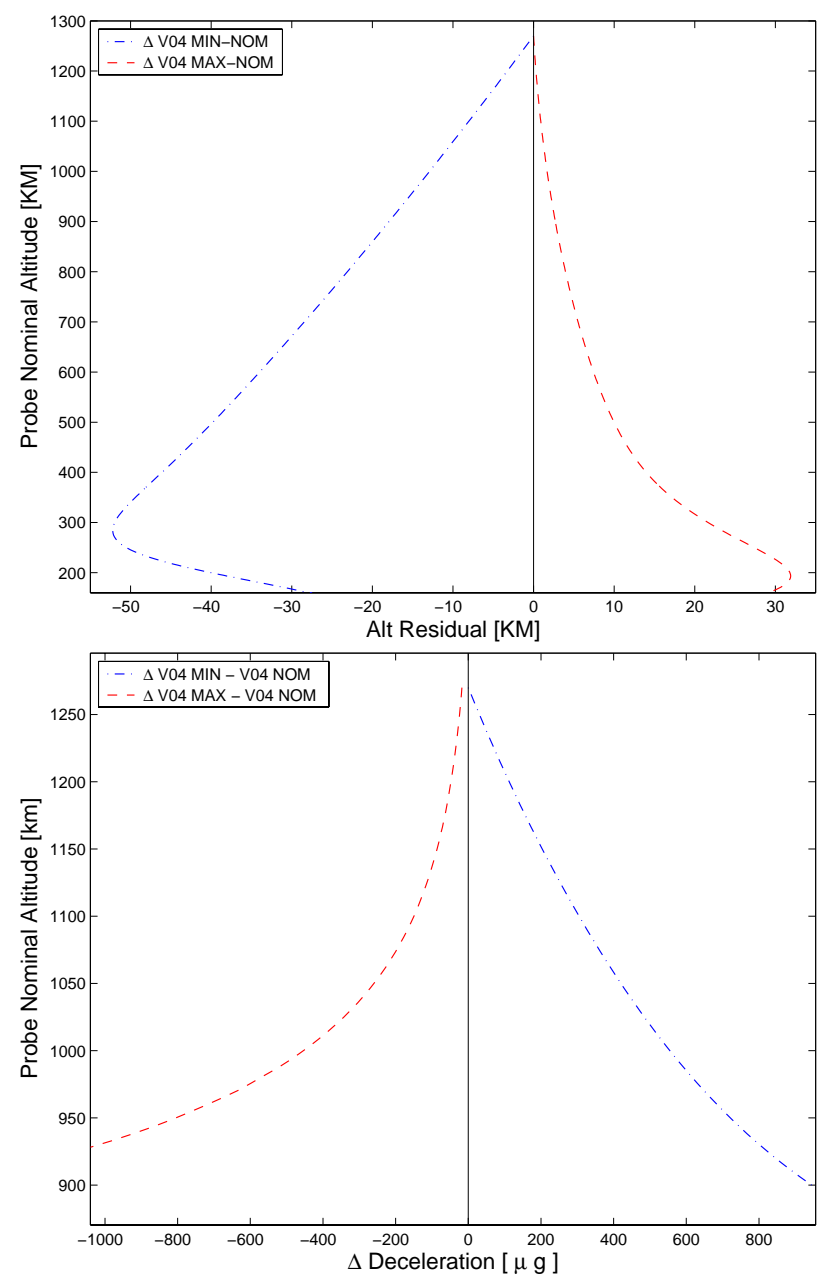

Fig. 3. Upper panel: difference in altitude between the Huygens trajectory simulations using the V04 minimum and maximum density profiles with respect to the V04 nominal one. Lower panel: difference in probe aerodynamic drag (expressed in Earth gravity units) for the upper part of the entry phase.

Mach 1.5 in just under five minutes (Clausen et al., 2002). Detailed simulations of the Huygens entry and descent trajectory were recently published by Kazeminejad et al. (2004) which are entirely based on the Y97 atmosphere model. The authors consider extreme case scenarios of parameters that drive the entry and descent mission sequence. The most important are the Titan atmosphere model in its three versions, i.e. minimum, recommended and maximum model, the probe entry angle $\gamma=-(65 \pm 3)^{\circ}$, and the entry and descent drag coefficient with a $1 \sigma$ error of $5 \%$. As our aim was to evaluate the impact of different atmosphere models on the probe trajectory, we constrained ourselves to the nominal entry conditions (i.e. $\gamma=-65^{\circ}$ ) and drag coefficients.

Our first goal was to simulate the probe entry trajectory using the V04 maximum density profile (this is the only one that exceeds the Y97 maximum envelope, as shown in the lower panel of Fig. 2) and to compare it to the corresponding trajectory based on the Y97 maximum profile. Our results show that the higher V04 density profile implies a minor trajectory deviation (up to $\sim 0.67 \mathrm{~km}$ at an altitude of $340 \mathrm{~km}$ ) with respect to the Y97 (maximum) simulation. This corresponds to a delay of $\sim 0.18 \mathrm{~s}$ in the probe parachute sequence initiation time, which is defined as $T_{0}$. Note that $T_{0}$ is scheduled exactly $6.375 \mathrm{~s}$ after the detection of the limit deceleration of $10 \mathrm{~m} \mathrm{~s}^{-2}$ on the trailing edge of the deceleration pulse.

Our second goal was to evaluate the aerodynamic drag forces and corresponding trajectories using the V04 minimum and maximum model and to compare them to the corresponding values obtained from the V04 nominal model. The upper panel of Fig. 3 shows the altitude residuals (i.e. difference between the min/max and the nominal V04 model) vs. the nominal trajectory altitude profile. One can see that the different densities of the two extreme profiles produce a deviation of up to $\sim 50 \mathrm{~km}$ for the minimum and $\sim 30 \mathrm{~km}$ for the maximum V04 profile. The nominal $T_{0}$ time for the V04 model is 4.57 min past interface epoch (the defined epoch at which the probe is at an altitude of $1270 \mathrm{~km}$ ) and occurs at an altitude of $\sim 158 \mathrm{~km}$. These values correspond very well to the corresponding values published for the $\mathrm{Y} 97$ model by Kazeminejad et al. (2004). The V04 extreme models introduce time shifts (with respect to the nominal epoch) to the parachute sequence starting time $\left(T_{0}\right)$ of $\sim+4.4 \mathrm{~s}$ for the minimum ( $T_{0}$ occurs at $\sim 133.9 \mathrm{~km}$ altitude) and $-12.4 \mathrm{~s}\left(T_{0}\right.$ occurs at $\sim 185.4 \mathrm{~km}$ altitude) for the maximum model.

The lower panel of Fig. 3 depicts the differences in aerodynamic drag for the minimum and maximum V04 density profiles with respect to the nominal V04 profile.

The Huygens Atmospheric Structure Instrument (HASI) (Fulchignoni et al., 2002) comprises one servo and one piezo accelerometer, which are aligned to the principal axes of the probe. The HASI accelerometers will be the only scientific instrument switched on during the high speed entry phase, which is characterized by a very wide range of accelerations from the limiting resolution of around $0.3 \mu \mathrm{g}$ up to a maximum of more than $12 \mathrm{~g}$. The effective sampling rate will be $3.125 \mathrm{~Hz}$ (Zarnecki et al., 2004). Assuming a probe entry mass of $320 \mathrm{~kg}$, a free molecular flow drag coefficient of $C_{D}=2.09$ (Thierry Blanquaert (ESA/ESTEC), private communication), and a crosssectional area of $A=5.73 \mathrm{~m}^{2}$ (Clausen et al., 2002) one obtains a limit density of $\rho \sim 4.2834 \times 10^{-15} \mathrm{~g} / \mathrm{cm}^{3}$. This limit is shown as a vertical line, together with the V04 and Y97 density profiles, in Fig. 4. One can see that the density detection limit is at an altitude of $\sim 1050$ and $1100 \mathrm{~km}$ for the Y97 and V04 minimum density profiles respectively. For the recommended version the detection altitude is even higher than $1350 \mathrm{~km}$. The limit altitude is for all three versions of the V04 and the Y97 models above the altitude range where temperature variations due to plasma interaction are expected to take place (see Sect. 2). 


\section{Cassini low Titan flyby simulation}

The Huygens probe mission occured during the Cassini Tc flyby at an altitude of $60,000 \mathrm{~km}$ on 14 January 2005 . In the 040622 reference trajectory (the baseline trajectory at the time this study was initiated) the subsequent flyby (i.e. T3) on 15 February 2005 had a planned altitude of only $1000 \mathrm{~km}$, which would have navigated Cassini through the upper layers of Titan's atmosphere (Strange, 2002, and private communication). Our initial low altitude flyby study was therefore done for the T3 flyby.

By the time the review comments were sent to the authors, two new reference trajectories had, in the meantime, been provided by the Cassini Navigation team, i.e. the 041001 and the 041001 reference trajectories (Strange, 2005). The release of the 041001 trajectory was motivated by concerns that uncertainties in the Iapetus $G M$ would cause risk to the Huygens probe mission (the distance of the probe's Iapetus flyby was therefore increased from $62282 \mathrm{~km}$ to $121128 \mathrm{~km}$ ). This was done by lowering $\mathrm{Tb}$ from $2197 \mathrm{~km}$ to $1200 \mathrm{~km}$ and raising T3 from $1000 \mathrm{~km}$ to $1577 \mathrm{~km}$. During the Ta flyby, Cassini experienced a higher thruster duty cycle than expected and due to concerns of spacecraft tumbling at low altitudes the first two $950 \mathrm{~km}$ flybys ( $\mathrm{T} 5$ and T7) were raised. The first $950 \mathrm{~km}$ targeted flyby in the 041210 trajectory is currently T16 on 22 July 2006; see Table 1 in Strange (2005).

The aerodynamic drag that Cassini will experience from Titan's upper atmosphere will have to be compensated for by the spacecraft attitude and articulation control subsystem (AACS), in order to avoid Cassini tumbling out of control. The per-axis atmospheric torque $M_{a}$ acting on the spacecraft can be derived from (Lee, 2002)

$M_{a}=\frac{1}{2} C_{D} \rho V_{r e l}^{2} A(c p-c m)$,

where $C_{D}$ is the drag coefficient which was estimated for the Cassini spacecraft in a free molecular flow field as $C_{D}=2.1 \pm 0.1$ (Lee, 2004). $\rho$ is the atmospheric density and $V_{r e l}$ is the spacecraft relative velocity with respect to Titan's atmosphere (taking into account the planet's rotation and an assumed constant velocity of $140 \mathrm{~m} / \mathrm{s}$ for Titan's high altitude prograde winds). $A(c p-c m)$ is the product of the projected area and offset of the center of pressure with respect to the center of mass. The $c p-c m$ depends on the orientation of the spacecraft during the flyby and can take the following values about the $\mathrm{Y}$-axis and $\mathrm{Z}$-axis, depending on the spacecraft orientation: 0.829 and $0.985 \mathrm{~m}^{2}$ for $\mathrm{ORS}^{2}$ to Titan and INMS $^{3}$ along ram orientation (BMX configuration), 1.113 and $0.140 \mathrm{~m}$ for ORS to Titan and $\mathrm{HGA}^{4}$ along ram orientation (BMZ configuration), and 0.829 and $0.985 \mathrm{~m}$ for HGA to Titan and spacecraft + Y-axis perpendicular to ram direction (BMX configuration), respectively. The projected area

\footnotetext{
${ }^{2}$ Optical Remote Sensing Pallet

${ }^{3}$ Ion and Neutral Mass Spectrometer

${ }^{4}$ High Gain Antenna
}

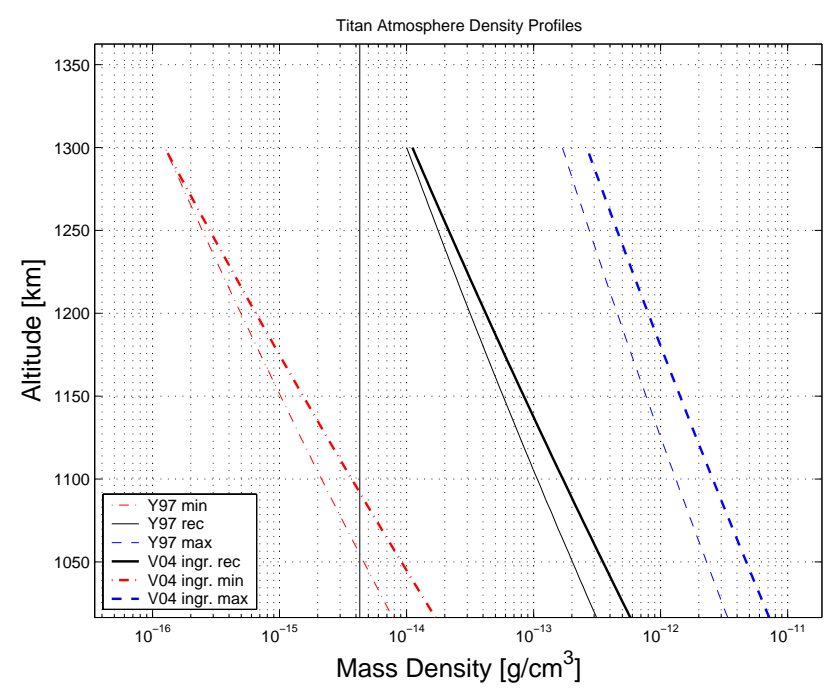

Fig. 4. HASI servo accelerometer detection limit for the three versions of the V04 and the Y97 atmosphere profiles. The vertical line shows the density detection limit of the HASI-ACC servo accelerometer

was assumed as $A=16.396 \mathrm{~m}^{2}$ for the BMX and $A=15.48 \mathrm{~m}^{2}$ for the BMZ configuration (Price, 1992).

We numerically integrated Cassini's T16 flyby trajectory using initial conditions from the Cassini reference trajectory kernel $^{5}$. Our simulation included gravitational forces from Saturn, Titan and the Sun, as well as aerodynamic drag from the V04 and Y97 minimum, recommended, and maximum density profiles. We used a spacecraft mass of $2986.8 \mathrm{~kg}$, according to Seal (2001). From the integrated spacecraft velocity vector we derived the per-axis atmospheric torque acting about Cassini's Z- and Y-axis for the BMX configuration. As expected we obtained the maximum torque at an altitude of $1000 \mathrm{~km}$ for the V04 maximum model with values of $\sim 4.5 \mathrm{Nm}$ and $\sim 5.4 \mathrm{Nm}$, respectively, about the Y- and $\mathrm{Z}$-axis. Figure 5 summarizes the $\mathrm{Z}$-axis torque profiles for the various atmosphere models. One can see that the V04 torque values are more than twice as high as the Y97 results. Comparing our results with the Cassini AACS worst case estimations from Lee (2000) and Lee (2002) we see that our worst case estimations are more than 5 times higher for the V04 maximum profile and more than 2.5 times higher for the Y97 maximum profile. Our simulations also showed that the V04 maximum atmosphere implies a minor (up to $\sim 150 \mathrm{~m}$ ) deviation of Cassini's trajectory from the reference trajectory (which does not take into account any aerodynamic drag).

Equation (1) provides a means to infer the atmospheric density (and with the assumption of hydrostatic equilibrium the pressure and temperature profiles) from the measured torque according to Eq. (1). This was done for the Ta flyby when the Cassini spacecraft experienced a higher thruster duty cycle than expected (Lee, 2004). The inferred density profile was considerably larger than the one derived from

\footnotetext{
${ }^{5}$ NAIF trajectory kernel: 041210AP_SCPSE_04329_08189.bsp
} 


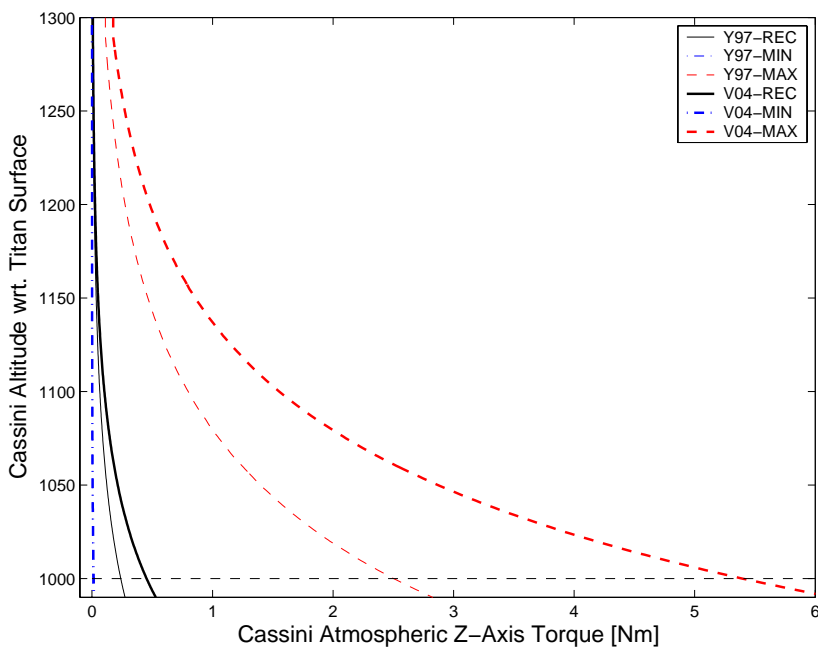

Fig. 5. Simulated atmospheric torque about Cassini Z-axis during T16 low altitude flyby on 22 July 2006, 00:25:27 UTC (041 210 reference trajectory) for the Y 97 and V04 recommended, minimum, and maximum density profiles; assumptions: projected area $=16.396$ $\mathrm{m}^{2}, C_{D}=2.2, c_{p}-c_{m}$ distance $=0.985 \mathrm{~m}$, BMX configuration.

orbiter instrument measurements such as the Ion and Neutral Mass Spectrometer (INMS). The uncertainty of the AACS inferred atmosphere density profile stems mainly from the combination of uncertainties of the drag coefficient $C_{D}$, the location of the center of pressure and mass, and the projected spacecraft area. From those the error on the reconstructed density profile was estimated by Lee (2004) to be in the range of $15-20 \%$.

\section{Concluding remarks}

Titan's upper atmosphere temperature profile is not only shaped by solar XUV radiation and magnetospheric electrons but can also be influenced by magnetospheric $\mathrm{N}^{+}$ions and high altitude monomer haze particles. We have incorporated these heating effects as temperature uncertainties into the V04 model and introduced two extreme profiles with a constant temperature offset from the nominal (published) one. We then derived corresponding density profiles and used them in our numerical trajectory simulation for both the Huygens entry trajectory and the Cassini low flyby trajectory at T16.

Our simulations show that the upper atmospheric temperature profiles will have an impact on the upper altitude limit at which the HASI servo accelerometer will be able to detect the atmosphere. This upper altitude limit can range from more than $1350 \mathrm{~km}$ down to only $1050 \mathrm{~km}$. The two extreme temperature profiles that were considered in our study leave a clear signature in both the integrated probe entry phase trajectory, as well as the aerodynamic drag force which will be measured by the HASI instrument aboard Huygens. Those measurements will be used to reconstruct the upper atmospheric properties of Titan and are therefore an important means to study the outlined atmospheric heating processes. One has to keep in mind, however, that this inversion depends on input parameters like the probe drag coefficient (currently with an uncertainty of 5\%), the atmospheric composition (this will only be measured during the probe descent phase), and the probe attitude (i.e. the angle-of-attack). A comparison of probe results with corresponding results as derived from Cassini instruments is therefore very important.

Our simulation results for the Cassini T16 low flyby provided us with a fairly accurate estimation of the atmospheric torque on the Cassini spacecraft. We obtained torque values for the Cassini Z-, and Y-axis that are twice as high for the newly introduced V04 maximum profile with respect to the Y97 corresponding model, and 4 times as high as the worst case estimation from Lee (2000) and Lee (2002).

We point out the possibility to infer an atmospheric density profile of Titan's upper atmosphere from the measured Cassini torque values, which will be provided by the AACS subsystem. This method was applied for the Cassini Ta flyby torque measurements and provided density profiles with a non negligible error bar (i.e. on the order of magnitude of $\pm 15 \%$, due to the uncertainties of input parameters) (Lee, 2004). We still encourage to apply this method to future Titan low flyby trajectories. This would not only provide additional density, pressure, and temperature profiles to validate the Huygens and INMS results but also complementary profiles, which could help to reveal the latitudinal and diurnal behaviours of Titan's upper atmospheric temperatures.

Acknowledgements. This study was supported by the International Space Science Institute (ISSI) and carried out in the frame of the ISSI Team "Titan a planetary scale laboratory in the year 2004". The authors also thank the European Space Agency's Research and Scientific Support Department for support via the Huygens project.

Topical Editor U.-P. Hoppe thanks I. Müller-Wodarg and another referee for their help in evaluating this paper.

\section{References}

Chassefière, E. and Cabane, M.: Two formation regions for Titan's hazes: indirect clues and possible synthesis mechanisms, Planet. Space Sci., 43, 91-103, 1995.

Clausen, K. C., Hassan, H., Verdant, M., Couzin, P., Huttin, G., Brisson, M., Sollazzo, C., and Lebreton, J.-P.: The Huygens Probe System Design, Space Sci. R., 104, 155-189, 2002.

Coustenis, A. and Bezard, B.: Titan's atmosphere from Voyager infrared observations. IV: Latitudinal variations of temperature and composition, Icarus, 115, 126-140, 1995.

Coustenis, A., Bezard, B., and Gautier, D.: Titan's atmosphere from Voyager infrared observations. I - The gas composition of Titan's equatorial region, Icarus, 80, 54-76, 1989.

Friedson, A. J. and Yung, Y. L.: The thermosphere of Titan, J. Geophys. Res., 89, 85-90, 1984.

Fulchignoni, M., Ferri, F., Angrilli, F., Bar-Nun, A., Barucci, M. A., Bianchini, G., Borucki, W., Coradini, M., Coustenis, A., Falkner, P., Flamini, E., Grard, R., Hamelin, M., Harri, A. M., Leppelmeier, G. W., Lopez-Moreno, J. J., McDonnell, J. A. M., McKay, C. P., Neubauer, F. H., Pedersen, A., Picardi, G., Pirronello, V., Rodrigo, R., Schwingenschuh, K., Seiff, A., Sved- 
hem, H., Vanzani, V., and Zarnecki, J.: The Characterisation of Titan's Atmospheric Physical Properties by the Huygens Atmospheric Structure Instrument (HASI), Space Sci. R., 104, 397434, 2002.

Hartle, R. E., Sittler, E. C., Ogilvie, K. W., Scudder, J. D., Lazarus, A. J., and Atreya, S. K.: Titan's ion exosphere observed from Voyager 1, J. Geophys. Res., 87, 1383-1394, 1982.

Kazeminejad, B., Pérez-Ayúcar, M., Lebreton, J.-P., SanchezNogales, M., Belló-Mora, M., Strange, N., Roth, D., Popken, L., Clausen, K., and Couzin, P.: Simulation and analysis of the revised Huygens probe entry and descent trajectory and radio link modelling, Planet. Space Sci., 52, 799-814, 2004.

Lammer, H. and Bauer, S. J.: Atmospheric mass loss from Titan by sputtering, Planet. Space Sci., 41, 657-663, 1993.

Lammer, H. and Stumptner, W.: High altitude haze: Influence of monomer particles on Titan's temperature profile, Planet. Space Sci., 47, 1341-1346, 1999.

Lammer, H., Stumptner, W., and Bauer, S. J.: Dynamic escape of H from Titan as consequence of sputtering induced heating, Planet. Space Sci., 46, 1207-1213, 1998.

Lebreton, J.-P. and Matson, D. L.: The Huygens Probe: Science, Payload and Mission Overview, Space Sci. R., 104, 59-100, 2002.

Lee, A.: Reconstruction of Titan Atmospheric Density Using Spacecraft AACS Flight Data, Presentation given at the Titan A Workshop, November 15, 2004, Tech. Rep., Cassini Spacecraft Operations Office at NASA-JPL, 2004.

Lee, Y. L.: FIRST Titan flyby - Three AACS Issues, Tech. Rep. IOM SCO-00-041, NASA-JPL, 2000.

Lee, Y. L.: Safe Titan Flyby Altitude on Reaction Wheels, Tech. Rep. IOM SCO-02-017, NASA-JPL, 2002.

Lellouch, E. and Hunten, D. M.: Titan atmosphere engineering model, Tech. Rep. ESLAB 87/199, ESA Space Science Department, 1987.

Lellouch, E., Coustenis, A., Gautier, D., Raulin, F., Dubouloz, N., and Frere, C.: Titan's atmosphere and hypothesized ocean - A reanalysis of the Voyager 1 radio-occultation and IRIS 7.7-micron data, Icarus, 79, 328-349, 1989.

Lellouch, E., Hunten, D. M., Kockarts, G., and Coustenis, A.: Titan's thermosphere profile, Icarus, 83, 308-324, 1990.

Lindal, G. F., Wood, G. E., Hotz, H. B., Sweetnam, D. N., Eshleman, V. R., and Tyler, G. L.: The atmosphere of Titan - an analysis of the Voyager 1 radio occultation measurements, Icarus, 53, 348-363, 1983.
Müller-Wodarg, I. C. F. and Yelle, R. V.: The effect of dynamics on the composition of Titan's upper atmosphere, Geophys. Res. L., 29, 54-1, 2002.

Neubauer, F. M., Gurnett, D. A., Scudder, J. D., and Hartle, R. E. Titan's magnetospheric interaction, 760-787, Saturn, 1984.

Price, H.: Cassini Projected area Plots, Tech. Rep. IOM 3522-92216, NASA-JPL, 1992.

Seal, D.: Cassini Mission Plan, Rev. M, Tech. Rep. PD- 699-100 Rev. M, Table 4.2, NASA-JPL, 2001.

Shematovich, V. I., Johnson, R. E., Michael, M., and Luhmann, J. G.: Nitrogen loss from Titan, J. Geo. Res. (Planets), 108, 6-1, 2003.

Smith, G. R., Strobel, D. F., Broadfoot, A. L., Sandel, B. R., Shemansky, D. E., and Holberg, J. B.: Titan's upper atmosphere Composition and temperature from the EUV solar occultation results, J. Geo. Res., 87, 1351-1359, 1982.

Strange, N.: Cassini Tour Redesign for the Huygens Mission, AIAA/AAS Astrodynamics Specialist Conference Paper, Monterey, CA., 2002-4720, 2002.

Strange, N.: Cassini 041001 and 041210 Refernce Trajectory Updates, Tech. Rep. 343M-05-001, NASA Jet Propulsion Laboratory, 2005.

Strobel, D. F., Summers, M. E., and Zhu, X.: Titan's upper atmosphere - Structure and ultraviolet emissions, Icarus, 100, 512526, 1992.

Vervack, R. J., Sandel, B. R., and Strobel, D. F.: New perspectives on Titan's upper atmosphere from a reanalysis of the Voyager 1 UVS solar occultations, Icarus, 170, 91-112, 2004.

Wolf, D. A. and Neubauer, F. M.: Titan's highly variable plasma environment, J. Geophys. Res., 87, 881-885, 1982.

Yelle, R. V.: Non-LTE models of Titan's upper atmosphere, Astrophys. J., 383, 380-400, 1991.

Yelle, R. V., Strobel, D. F., Lellouch, E., and Gautier, D.: The Yelle Titan Atmosphere Engineering Models, in ESA SP-1177: Huygens: Science, Payload and Mission, 243, 1997.

Zarnecki, J. C., Ferri, F., Hathi, B., Leese, M. R., Ball, A. J., Colombatti, G., and Fulchignoni, M.: In-flight performance of the HASI servo accelerometer and implications for results at Titan, in ESA SP-544: Planetary Probe Atmospheric Entry and Descent Trajectory Analysis and Science, 71-76, 2004. 